\title{
Current State and Future Perspectives of Nanotechnology In Dentistry
}

\author{
Yogesh Upadhyay \\ Chandra Dental College And Hospital.,Safedabad Barabanki.U.P. India
}

\begin{abstract}
SUMMARY : Nanotechnology has been considered as multidisciplinary field of scientific research about different types of nanoparticles as well as the application of new nanomaterials and nanodevices in numerous areas of human interest. It offers advances in industry, engineering, information and communication technology, electronics, environmental science and energy savings, economics etc. New nanoproducts and nanotehnology could be applied in almost all fields of human activity. Potential benefit of nanomaterials and nanorobots applied in medicine and dentistry is of main concern when thinking about nanoadvances. However, nanotechnology has become a controversial issue between scientific and public opinions due to the insufficient knowledge of potential hazard to human health and environment. Some of the raised questions are what are the advantages and disadvantages of nanotechnological evolution, and what kind of future can be expected when changes gain wider scale? The aim of this study was to present the importance of nanotechnology in various areas, especially in medicine and dentistry, and to point out possible consequences of their use to human health and environment.
\end{abstract}

KEYWORDS: nanotechnology; nanomaterials; dentistry

\section{INTRODUCTION}

The universal definition of nanotechnology has not been established yet, since the existing definitions vary depending on the intended use. Therefore, current attempts to define nanotechnology can be divided in the three main groups: scientific definitions, public definitions and those that allow making certain decisions [1]. Nanotechnology is the science and engineering involved in design, synthesis, characterization and application of materials and devices whose smallest unit of measurement in at least one dimension is on the nanometer scale (one billionth of a meter, 10-9 m) [2]. This means that what we consider nanomaterial or a nanodevice, has the smallest dimension of about $100 \mathrm{~nm}$ i.e. maximum $1 \times 10-7 \mathrm{~m}$. For comparison, the diameter of human hair is about $50 \mu \mathrm{m}$ and it is 50,000 times larger than the size of $1 \mathrm{~nm}$ [3]. Further, the average diameter of the bacterium Streptococcus mutans 10449 is around $0.321 \pm 0.007 \mu \mathrm{m}$ [4], while the size of a hydrogen atom is in the range of 0.1 to $0.2 \mathrm{~nm}$ [5]. Nanotechnology and the science of nanomaterials have a potential to provide benefits in numerous areas such as synthesis of new materials with advanced properties, production technology, information technology and elec- tronics, ecology and energy conservation, nanobiosys- tems, medical appliances, transportation, economy, etc. It is unlikely that there is any segment of human life and activity in which nanotechnology cannot be applied. It is also estimated that nanotechnology will affect the psychology of people as well as their understanding of the world they live in (social phenomena, philosophical views and ethical considerations) $[6,7,8]$. According to the U.S. National Institutes of Health, nanomedicine includes the application of nanotechnology in a "very specific medical intervention at the molecular level, i.e. to treat disease and repair damaged tissue such as bones, muscles and nerves." Johnson and McGee [9] maybe defined nanomedicine more appropriate as "nanomedicine involves medical interventions that use materials, machines and mechanisms created or controlled by nanoscience and nanotechnology in order to monitor, improve or maintain health". Accordingly, the potential applications of nanotechnology in medicine are drug and genetic material delivery (viral vectors, non-viral vectors, such as nanoparticles, liposomes, or dendrimers and so-called "gene guns"), imaging, molecular diagnosis, therapy, cardiology, orthopedics, etc. [2, 10-13]. Most of nanotechnology applied in medicine, can also be applied in dentistry. Nanodentistry has a potential to improve oral health by providing sophisticated preventive, diagnostic and therapeutic measures using nanomaterials, biotechnology and nanorobots [14]. That way, advanced dental care could be delivered throughout the world population $[2,15]$. The structure of material that has particles of a nanometer size possesses special properties. Physical and chemical properties of materials become quite different when number of constitutional atoms is greatly reduced. A small number of atoms allow different positioning and distance between them making those properties to be dominant physical and chemical properties of the object $[5,16]$. 


\section{EARLY VISION}

At the annual meeting of the American Physical Society, back in 1959, the physicist and Nobel laureate Richard P. Feynman has drawn great attention of the scientific community by his revolutionary statement that "there is a plenty of room at the bottom." He talked about future possibility to create materials and devices at the atomic or molecular level and by his speech he has influenced human mind to conceive an innovative way of thinking. He suggested that the existing machinery and equipment should produce smaller tools which would than produce even smaller machines and tools and so on, up to the molecular level. He suggested that nanomachines, nanorobots and nanodevices could be ultimately used to develop a wide range of atomically precise microscopic instruments and production tools. Its historical lecture Feyman concluded with words: "This is a developmental path that cannot be avoided " $[17,18]$.

\section{CURRENT STATE AND FUTURE PERSPECTIVES IN DENTISTRY}

Nanotechnology offers a broad range of innovations and improvements in prevention, diagnostics and treatment of oral diseases. The characteristics of composite filling materials have been constantly improved. The mode for producing filler particles for composite materials has been changed on nanotehnological level in order to produce particles smaller than $100 \mathrm{~nm}$. Traditional process for the production of small particles has been character- ized by grinding larger pieces of glass, quartz or ceramics, i.e. approach "from the top to the bottom" ("top-down" approach). In order to produce particles smaller than $100 \mathrm{~nm}$, that method has been replaced by chemical processes of direct molecular synthesis and bonding (direct molecular assembly) i.e. applying the approach "from the bottom to the top" ("bottom up" approach). Nanofilled composite systems have shown better physical properties including compressive strength, tensile strength, impact strength, flexural strength and abrasion resistance, etc. than traditional composites filled with microparticles [5]. In addition, aesthetic properties of nanomaterials are superior as compared to traditional, primarily due to the optical properties of nanoparticles as well as better polishability and maintaining polished surface for long time [5]. There are two main types of dental composites filled with nano- fillers: nanohybrids and nanofilled. Nanohybrid composites contain fillers of different size e.g. larger glass particles (average size about $2000 \mathrm{~nm}$ ) mixed with particles of a size around $10 \mathrm{~nm}$, while nanofilled have more uniform particle size e.g. particle size of about $75 \mathrm{~nm}$ mixed with particles of sizes from 5 to $25 \mathrm{~nm}$. Nanofilled composite materials are characterized by the combination of nanoparticles and "nano-cloud" (nanoclusters) introduced in Filtek Supreme (3M ESPE). These materials exhibit improved mechanical and physical properties as compared to the conventional composite materials for dental fillings [19]. In the future, nanotechnology and biomimetic approach (biomimetics - mimicking the natural structure and properties of biological materials), could be used for repair and restoration of damaged enamel [20]. Chen et al. [21] have synthesized hydroxyapatite nano-rods which have a structure similar to the enamel prisms (enamel- prism-like hydroxyapatite nanorods). These hydroxyapatite nano-rods possess a feature so called self-connection and switching (self-assembly) and may become nano- restoration that mimic naturally occurring processes, and as such, could be used to restore tooth structure. Similar nano-structures that could also be used for the restoration of tooth substance are nanospheres; genetically engineered peptides that bind to the surface of inorganic materials (GEPIs - genetically engineered peptides for inorganics), amelogenin-based materials as well as restorations that release fluoride [19, 22, 23].

There are number of published papers that have studied possibilities for dental caries prevention of using the benefits of nanotechnology [20, 24-27]. In this studies, nanomaterials have been synthesized and tested. For example, biphasic calcium fluoride/fluorinated hydroxya- patite (CF/FHAp) nanocrystals have been synthesized, characterised and in vitro tested as potential dental filling material with ability to prevent dental caries by fluoride ion release [24]. Also, Min et al. [25] in an in vitro study presented the application of hydroxyapatite nanoparticles which, if added to soft drinks for athletes, could prevent teeth erosion. Furthermore, Kim et al. [26], in laboratory conditions, tested the effect of nano-carbonate apatite (n-CAP) in the prevention of late discoloration of teeth after bleaching. They found that $10 \% \mathrm{n}$-CAP can substan- tially maintain achieved color after whitening and provide adequate recovery of tooth enamel. Based on the current knowledge, it is certain that nanotechnology has a great potential for prevention of dental caries. Tschoppe et al. [27] found in their study that toothpaste and preparations for the caries prevention that contain nanohydroxyapatite (n-HAp) can enhance the process of remineralization in enamel and dentin. In an in vitro study, they compared the effect of toothpaste with added n-HAp or zinc-carbonate/nanohydroxyapa- tite ( $\mathrm{ZnCO} 3 / \mathrm{n}$ Hap) particles with conventional fluoride toothpastes on bovine enamel and dentin remineralization. They found similar or better effects when used tooth- paste with nanoparticles [27].In the sphere of dental implantology that is making steady progress, further improvement is possible by modifying the surface of endosseous implants with nanoscale surface modification techniques. Such altered properties of nanosurfaces affect cell adhesion, proliferation, and differentiation. The process of osseointegration could be better and faster using that type of surface modification. 
There are already a few commercial nano-modified dental implant systems available for clinical use [28]. Time will show strengths and weaknesses of dental implants surface modifications through long-term clinical evaluation.Some promising results using nanotechnology modification have already been obtained in one of the most popular areas in dentistry, guided tissue and periodontal regeneration [29]. New materials with addition of nano- particles should serve as a scaffold for tissue ingrowths to improve the ability of tissue regeneration. Srinivasan et al. [29] found that experimental alginate/nano-bioactive glass ceramic composite scaffolds could be useful in periodontal regeneration. Such structure provides good protein adsorption and cell adhesion and proliferation. Although modern protocols for the treatment of pulp diseases and apical periodontitis guarantee high success rates, a new approach in endodontic therapy, which would be also the ideal therapy, is the induction of healthy tissue and replacment of diseased or necrotic pulp tissue. Fioretti et al. studied the anti-inflammatory effects of biologically active nanostructured multilayer films on fibroblasts [30]. They reported first time use of these films containing melanocortin peptides $(\alpha-\mathrm{MSH})$, (which can stimulate human pulp fibroblasts in order to modulate pulpal inflamation) as a new active biomaterial for endodontic regeneration. If the idea of nanorobots becomes realized, they would have a significant application in orthodontics. Manipulation with periodontal tissue would allow teeth movement and positioning [18]. Natural tooth appearance and durability, new forms of local anesthesia, treatment of dentine hypersensitivity, regeneration of tooth structure, improving preventive and regenerative procedures are also some areas for potential use of these nano-systems $[2,17,18]$.

\section{NANOTECHNOLOGY AS A RISK FOR HUMAN HEALTH AND ENVIRONMENT}

The amount of free nanoparticles in nature depends on various factors: their physico-chemical properties, quantity and time of exposure. Nanomaterials released in the enviroment can be further modified by: temperature, $\mathrm{pH}$, different biological conditions and presence of other pollutants. In this interaction nanomaterials can alter atmosphere, soil and water. These transformations and interactions can adversely affect the current state of the environment and be harmful to human health and balance of the ecosystems [31, 32, 33]. Nanoparticles have unique physicochemical properties that can be different in comparison with the same material in macroscale size. The main characteristic of these particles is greater difference between the surface area and unit weight, and higher surface reactivity $[3,4,15]$. It might lead to increased absorption through the lungs, skin, digestive tract and it might cause side effect to the lungs and other organs [2, 17]. Nanoparticles are so small that they can interact with DNA, RNA and other intracelular components. The question is what kind of immune response could be activated by these small particles, and what will be the metabolism, absorption and elimination path of these products? There is a need for systemic solutions, monitoring and recording of potential hazard as well as finding timely responses in order to achieve safety for human health and environment.

\section{CONCLUSION}

It can be predicted that nanotechnology will have a great impact on dental research, dental prevention, diagnostics and treatment solutions. Major advances are expected in the sphere of preventive dentistry and mimicking pro- cesses that occur in nature (biomimetics). More accurate diagnostics will lead to detecting diseases in the earliest stages allowing timely applied treatment. Nanotechnology will create better treatment solutions themselves, and thus, overall features of oral health care will be improved. On the other hand, there are poorly understood risks for human health and environment that nanotechnology might cause. What will the application of nanotechnol- ogy and nanomaterials bring to the world, remains to be seen. However, nanotechnogy will be in focus of modern civilization reflection and action.

\section{REFERENCES}

[1] Balogh L. Why do we have so many definitions for nanoscience and nanotechnology? Nanomed Nanotechnol Biol Med. 2010; 6:397-8

[2] Sahoo SK, Parveen S, Panda JJ. The present and future of nano- technology in human health care. Nanomed Nanotechnol Biol Med. 2007; 3:20-31.

[3] Jandt KD, Sigusch BW. Future perspectives of resin-based dental materials. Dent Mater. 2009; 25:1001-6.

[4] Ryan V, Hart TR, Schiller R. Size determination of streptococcus mutans 10449 by laser light scattering. Biophys J. 1980; 31:313-24.

[5] Mitra SB, Wu D, Holmes BN. An application of nanotechnology in advanced dental materials. JADA. 2003; 134:1382-90.

[6] Davies S. How we talk when we talk about nano: the future in lay- people's talk. Futures. 2011; 43:317-26.

[7] Burri R, Bellucci S. Public perception of nanotechnology. J Nanopart Res. 2008; 10:387-91.

[8] Kahan DM. Nanotechnology and society - the evolution of risk perceptions. Nature Nanotech. 2009; 4:705-6.

[9] Johnson S, McGee G. Nanotechnologies in health care: a needs assesment regarding ethics and policy in nanomedicine. Available from: http://www.hcs.harvard.edu/ hhpr/currentissue/07spring/156-164. pdf Published 2007, Accessed December 26th, 2011

[10] Emerich DF, Thanos CG. Nanotechnology and medicine. Expert Opin Biol Ther. 2003; 3:655-63.

[11] Moghimi SM, Hunter AC, Murray JC. Nanomedicine: current status and future prospects. FASEB J. 2005; 19:311-30.

[12] Emerich DF. Nanomedicine - prospective therapeutic and diagnostic applications. Expert Opin Biol Ther. 2005; 5:1-5.

[13] Jain KK. Nanodiagnostics: application of nanotechnology in molecular diagnostics. Expert Rev Mol Diagn. 2003; 3:153-61. 
[14] Subramani K, Ahmed W. Emerging Nanotechnologies in Dentistry: Processes, Materials and Applications. Waltham, MA: Elsevier Inc; 2012.

[15] Kanaparthy R, Kanaparthy A. The changing face of dentistry: nano- technology. Int J Nanomedicine. 2011; 6:2799-804.

[16] Trajković V, Marković Z. Nanomedicina: stanje i perspektive. In: Raković D, Uskoković D. Biomaterijali. Beograd: Institut tehničkih nauka SANU, Društvo za istraživanje materijala; 2010. p.762-77.

[17] Freitas RA Jr. Nanotechnology, nanomedicine and nanosurgery. Int J Surg. 2005; 3:243-6.

[18] Gupta J. Nanotechnology applications in medicine and dentistry. J Investig Clin Dent. 2011; 2:81-8.

[19] Saunders S. Current practicality of nanotechnology in dentistry. Part I: Focus on nanocomposite restoratives and biomimetics. Clin Cosmet Investig Dent. 2009; 1:47-61.

[20] Hannig M, Hannig C. Nanomaterials in preventive dentistry. Nature Nanotech. 2010; 5:565-9.

[21] Chen N, Clarkson BH, Sun K, Mansfield JF. Self-assembly of synthetic hydroxyapatite nanorods into enamel prism-like structure. J Colloid Interface Sci. 2005; 288:97-103.

[22] Zhou B, Liu Y, Wei W, Mao J. GEPIs-HA hybrid: a novel biomaterial for tooth repair. Med Hypotheses. 2008; 71:591-3.

[23] Tamerler C, Sarikaya M. Molecular biomimetics: nanotechnology and bionanotechnology using using genetically engineered pep- tides. Phil Trans R Soc A. 2009; 367:1705-26.

[24] Azami M, Jalilifiroozinezhad S, Mozafari M, Rabiee M. Synthesis and solubility of calcium fluoride/hydroxy-fluorapatite nanocrystals for dental applications. Ceram Int. 2011; 37:2007-14.

[25] Min J, Kwon H, Kim B. The addition of nanosized hydroxyapatite to a sports drink to inhibit dental erosion - in vitro study using bovine enamel. J Dent. 2011; 39:629-35.

[26] Kim Y, Kwon H, Kim B. Effect of nano-carbonate apatite to pre- vent re-stain after dental bleaching in vitro. J Dent. 2011; 39:636-42.

[27] Tschoppe P, Zandim D, Martus P, Kielbassa A. Enamel and den- tine remineralization by nano-hydroxyapatite toothpastes. J Dent. 2011; 39:430-7. 28. Mendonça G, Mendonça D, Aragão F, Cooper L. Advancing dental implant surface technology - from micron- to nanotopography. Biomater. 2008; 29:3822-35.

[28] Srinivasan S, Jayasree R, Chennazhi K, Nair S, Jayakumar R. Biocompatible alginate/nano bioactive glass ceramic composite scaffolds for periodontal tissue regeneration. Carbohyd Polym. 2012; 87:274-83.

[29] Fioretti F, Mendoza-Palomares C, Helms M, Al Alam D, Richert L, Arntz Y, et al. Nanostructured assemblies for dental applications. ACS Nano. 2010; 4:3277-87.

[30] National Nanotechnology Initiative, Environmental, Health and Safety Issues, Available from: http://www.nano.gov/you/environ- mental-health-safety. Accessed January 14th, 2012.

[31] Barnard AS. Nanohazards: knowledge is our first defence. Nature Mater. 2006; 5:245-8

[32] Haramina T. Rizici uporabe nanočestica. Polimeri. 2008; 29:115. 